\title{
Catalyst Design Using Nanoporous Iron for the Chemical Vapor Deposition Synthesis of Single-Walled Carbon Nanotubes
}

\author{
Tarek M. Abdel-Fattah, ${ }^{1}$ Phillip A. Williams, ${ }^{2}$ Russell A. Wincheski, ${ }^{2}$ and Qamar A. Shams ${ }^{3}$ \\ ${ }^{1}$ Applied Research Center, Thomas Jefferson National Accelerator Facility and Department of Molecular Biology and Chemistry, \\ Christopher Newport University, Newport News, VA 23606, USA \\ ${ }^{2}$ Nondestructive Evaluation Sciences Branch, NASA Langley Research Center, Hampton, VA 23681, USA \\ ${ }^{3}$ Aeronautics Systems Engineering Branch, NASA Langley Research Center, Hampton, VA 23681, USA
}

Correspondence should be addressed to Tarek M. Abdel-Fattah; fattah@cnu.edu

Received 15 March 2013; Accepted 18 September 2013

Academic Editor: Nadya Mason

Copyright (C) 2013 Tarek M. Abdel-Fattah et al. This is an open access article distributed under the Creative Commons Attribution License, which permits unrestricted use, distribution, and reproduction in any medium, provided the original work is properly cited.

\begin{abstract}
Single-walled carbon nanotubes (SWNTs) have been synthesized via a novel chemical vapor deposition (CVD) approach utilizing nanoporous, iron-supported catalysts. Stable aqueous dispersions of the CVD-grown nanotubes using an anionic surfactant were also obtained. The properties of the as-produced SWNTs were characterized through atomic force microscopy and Raman spectroscopy and compared with purified SWNTs produced via the high-pressure CO (HiPCO) method as a reference, and the nanotubes were observed with greater lengths than those of similarly processed HiPCO SWNTs.
\end{abstract}

\section{Introduction}

Of vital importance to the next generation of aerospace vehicles are structurally resilient, lightweight, and space durable materials and structural health monitoring sensors that can withstand environmental rigors. Carbon nanotubes (CNTs) have shown significant potential in these and a wide variety of other applications on the basis of their remarkable mechanical and electronic properties $[1,2]$. CNTs, however, can be produced by several synthesis methods, with some methods better suited for particular applications. Single-walled carbon nanotubes (SWNTs) grown between suspended pillars, for example, have been studied as potential nanoscale power lines [3]. A dominant approach for the synthesis of SWNTs is chemical vapor deposition (CVD), including the highpressure carbon monoxide (HiPCO) technique [4]. In this paper, we present an alternative CVD method of synthesis of SWNTs for use in composites and aerospace sensor applications. Specifically, we describe the novel incorporation of iron catalysts within a mesoporous material for CNT production.

Mesoporous materials (MPMs) are a class of inorganic molecular sieves [5]. Pores in the nanoscale range of 2 to $100 \mathrm{~nm}$ make these materials very attractive as shape selective adsorbents or catalysts. Generally, the MPM is prepared in solution by supramolecular assembly of organic molecules as templates with an inorganic precursor. In the original synthesis of MPMs is the formation of rod-like micelles using charged surfactants such as alkyltrimethylammonium surrounded by inorganic species followed by the polymerization of silicates to form the framework of MPMs. The pore size of MPMs can be tuned in the nanosize range based on the number of carbon atoms in the alkyl chain length of surfactants from about $1.6 \mathrm{~nm}$ for eight carbon atoms to $3.8 \mathrm{~nm}$ for 16 carbon atoms. The pores are separated by silicate walls whose thickness is in the range of 0.8 to $1.6 \mathrm{~nm}$. Assynthesized MPMs have specific crystalline phases that can be controlled by varying the ratio of surfactants to silicates. For example, MCM- 41 has independent channels; MCM-48 has cubic and interconnected channels, whereas MCM-50 has a layered structure. Organic templates can be removed by heating as-synthesized MPM without significant structural changes except for MCM-50, in which the structure collapses. After calcination, MCM-41 and MCM-48 materials have large surface area per gram ratios making them excellent candidates for pollutant remediation and catalysts for large molecules. For example, Abdel-Fattah et al. have synthesized 
close-ended multiwalled carbon nanotubes (MWNTs), in the absence of metal catalysts, from sucrose using mesoporous MCM-41 silicate as templates [6].

Varying the templates from charged surfactants to neutral surfactants such as primary amines produces hexagonal mesoporous silica materials (HMS) with thicker walls in the range of $2-3 \mathrm{~nm}[5,7]$. Introducing active metal centers in the framework makes HMS very appealing as catalysts for SWNT synthesis. In a previous study, Abdel-Fattah and Pinnavaia had demonstrated the introduction of different active centers, such as Sn and Fe, into the framework of HMS for ring opening polymerization of lactic acid [8]. Later on, other research studies had studied also the synthesis and the catalytic properties of Fe-HMS materials such as phenol hydroxylation $[9,10]$.

In this paper, we report the use of iron modified HMS (Fe-HMS) as catalysts for the synthesis of high-quality SWNTs by chemical vapor deposition. The as-produced nanotubes exhibit the ability for dispersion in aqueous surfactant solutions and for selective deposition on surfaces via dielectrophoresis. Material characterization is documented with atomic force microscopy and Raman spectroscopy and compared with HiPCO SWNTs as a reference.

\section{Experimental Details}

2.1. Catalyst Design and Preparation. Fe-HMS samples with Fe $2 \%$ were prepared with dodecylamine as a directing agent, as described in our previous work [8]. Briefly, the synthesis comprised the mixture of two solutions: one containing tetraethyl orthosilicate, iron (III) ethoxide, and another containing dodecylamine in water/ethanol mixture. After mixing, this solution was then stirred at room temperature for $24 \mathrm{~h}$. The solid, denoted Fe-HMS, was recovered by filtration, washed with hot water, and air-dried. Amines occluded in the mesopores were removed by calcination in air at $773 \mathrm{~K}$ for $4 \mathrm{~h}$.

2.2. CNT Growth. Carbon nanotubes were formed by CVD of methane at $1193 \mathrm{~K}$ on the $2 \% \mathrm{Fe}-\mathrm{HMS}(2 \% \mathrm{Fe})$, as catalysts. For a methane CVD experiment, $10 \mathrm{mg}$ of $2 \%$ Fe-HMS was placed in a quartz tube mounted in a tube furnace. An argon flow was passed through the quartz tube as the furnace was heated to reach $1193 \mathrm{~K}$. The Ar flow was then replaced by methane (99\% purity) at a flow rate of $1500 \mathrm{~cm}^{3} / \mathrm{min}$ under $1.25 \mathrm{~atm}$ head pressure. The methane flow lasted for $10 \mathrm{~min}$ and subsequently was replaced by argon, and the furnace was cooled to room temperature.

2.3. CNT Aqueous Surfactant Suspension and Dielectrophoresis. As-produced, raw CNT material was suspended in deionized water containing $0.5 \mathrm{wt} \%$ sodium dodecylbenzene sulfonate (NaDDBS), an anionic surfactant [9]. The aqueous suspension was subjected to a protocol, similar to that described by Paredes and Burghard [10], consisting of centrifugation followed by agitation in an ultrasonic bath to disperse the CNTs uniformly in the suspension. Immediately before deposition onto a substrate, the suspension was sonicated again for 25 minutes. As a reference, separate, aqueous NaDDBS suspensions of purified HiPCO SWNTs were also processed via the aforementioned protocol for comparison with CNTs synthesized via the Fe-HMS CVD method.

Substrates consisted of oxidized Si samples with metal electrodes first patterned via photolithography for large scale contacts and then followed by electron beam lithography for submicron features and contacts to the CNTs. Thin metal films of electrode pairs with $1.5 \mu \mathrm{m}$ spacing, patterned via electron beam lithography, were deposited via thermal evaporation of $\mathrm{Cr}$ as an adhesion layer followed by $\mathrm{Au}$ onto the substrates. CNTs were then deposited and aligned to the electrode pair locations through use of dielectrophoresis (DEP) $[11,12]$. For the dielectrophoretic deposition, an AC voltage of $10.0 \mathrm{~V}$ peak to peak with a frequency of $2.0 \mathrm{MHz}$ was applied between the $1.5 \mu \mathrm{m}$ gaps of the electrode pairs for 10 minutes as a droplet of the CNT suspension was placed on the samples.

2.4. Material Characterization and Analysis. 2\% Fe-HMS catalyst samples were characterized by X-ray diffraction (XRD) using a Philips PW 1710 diffractometer ( $\mathrm{Cu}$ Ka radiation). The nitrogen adsorption and desorption isotherms at $77.4 \mathrm{~K}$ were measured using a Coulter Omnisorp 360 CX sorptometer or ASAP 2010 sorptometer via a continuous adsorption procedure. Before the measurements, samples were evacuated overnight at $423 \mathrm{~K}$ and $10^{-6}$ Torr. The BET surface area was calculated from the linear part of the BET plot. The pore size distributions were calculated from adsorption branches of $\mathrm{N}_{2}$ adsorption/desorption isotherms based on the BJH model. Transmission electron microscopy (TEM) was performed to characterize the structure and morphology of the Fe-HMS catalysts material. Also, scanning electron microscopy (SEM) equipped with energy dispersive spectroscopy (EDS) was used to determine chemical composition of the Fe-HMS catalyst materials.

The physical and morphological properties of the CNTs were characterized via Raman spectroscopy and atomic force microscopy (AFM). Raman spectra were acquired with a Kaiser dispersive Raman spectrometer operating with an excitation laser of $785 \mathrm{~nm}$ wavelength. Topographic images from atomic force microscopy were obtained using a Digital Instruments-Veeco Metrology Group Nanoscope IIIa Multimode AFM in tapping mode. AFM analysis software was used for CNT diameter and length measurements within instrumental resolutions of $0.1 \mathrm{~nm}$ and $50 \mathrm{~nm}$, respectively. AFM image quality for samples of CNTs deposited from aqueous surfactant suspension onto patterned substrates was improved through the removal of excess surfactant on the samples by washing the samples with methanol and then heating them to $180^{\circ} \mathrm{C}$ in air for 2.5 hours, as has also been reported previously in other studies $[9,10]$.

\section{Results and Discussion}

The results of the preparation of the Fe-HMS catalysts were investigated via several techniques. Figure 1 shows the structure and morphology of a 2\% Fe-HMS sample analyzed by TEM. The TEM image qualitatively reveals the uniform periodic, porous wormhole structure of the 2\% Fe-HMS and the range of $25 \mathrm{~nm}$ similar to that already reported for pure silica HMS [7]. Quantitative analysis of the XRD data 
TABLE 1: Chemical composition of 2\% Fe-HMS catalyst via EDS.

\begin{tabular}{lcr}
\hline Element & Normalized weight \% & Atom \% \\
\hline $\mathrm{C}$ & $51.18 \pm 0.30$ & $60.84 \pm 0.36$ \\
$\mathrm{O}$ & $37.58 \pm 0.33$ & $33.53 \pm 0.29$ \\
$\mathrm{Al}$ & $0.22 \pm 0.01$ & $0.12 \pm 0.01$ \\
$\mathrm{Si}$ & $10.68 \pm 0.06$ & $5.43 \pm 0.03$ \\
$\mathrm{Fe}$ & $0.34 \pm 0.06$ & $0.09 \pm 0.01$ \\
\hline
\end{tabular}

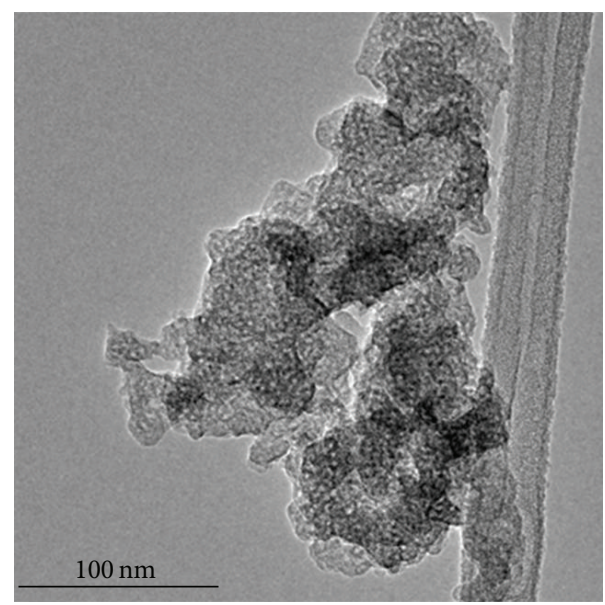

FIgure 1: TEM image of 2\% Fe-HMS catalysts.

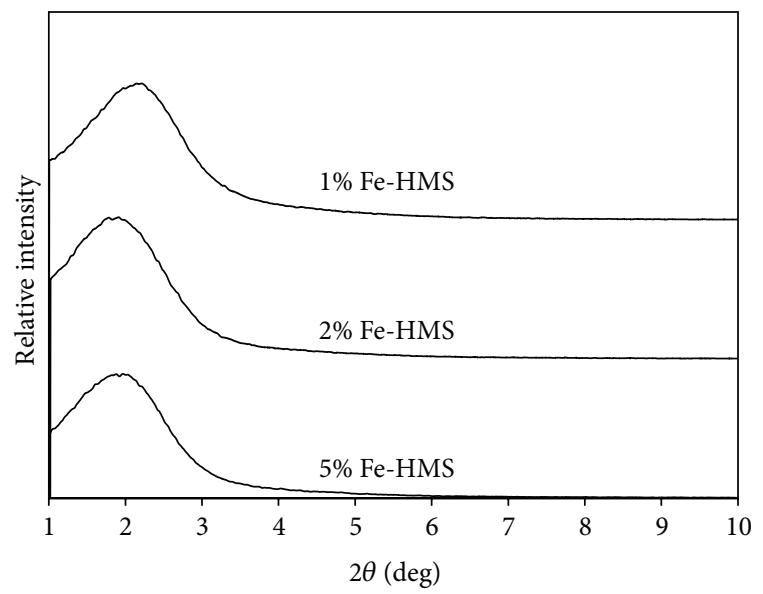

FIGURE 2: Powder XRD pattern of Fe-HMS samples with varying concentrations of Fe.

for the Fe-HMS materials, as shown in Figure 2, indicates an XRD pattern composed of a unique broad peak at $2.0^{\circ}$, similar to that already reported for pure silica HMS [7]. The $\mathrm{N}_{2}$ adsorption/desorption isotherms in Figure 3 show that the solids contain regular pores of ca. $2.7 \mathrm{~nm}$ diameter. Comparison with the X-ray powder data gives an average wall thickness of ca. $1.8 \mathrm{~nm}$, in agreement with previous determinations. Further, calculations of the BET surface area of the $2 \% \mathrm{Fe}-\mathrm{HMS}$ catalysts yield an average surface area of $980 \mathrm{~m}^{2} / \mathrm{g}$.

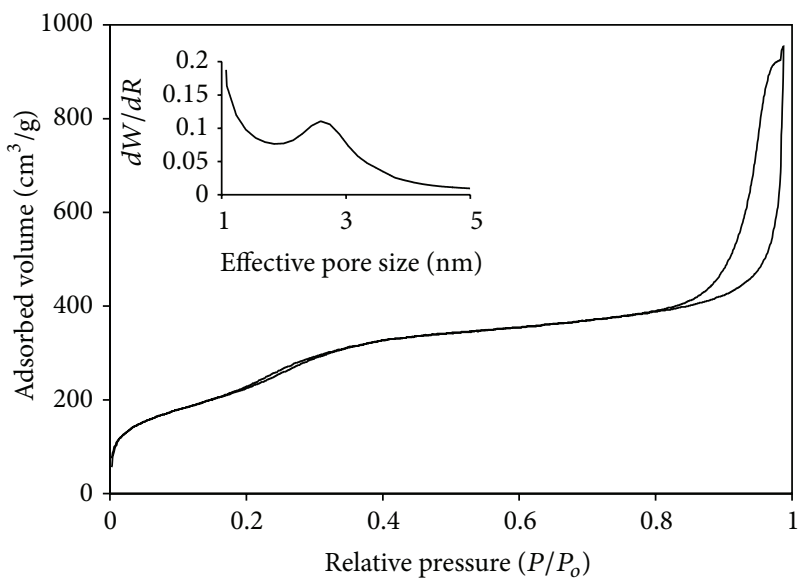

FIGURE 3: The nitrogen adsorption and desorption isotherms for $2 \% \mathrm{Fe}-\mathrm{HMS}$, measured at $77.4 \mathrm{~K}$. The inset presents the HorvathKawazoe pore size distribution curve for the Fe-HMS catalysts, where $d W / d R$ is the derivative of the normalized $\mathrm{N}_{2}$ volume adsorbed with respect to the pore size (diameter) of the adsorbent.

To characterize the elemental chemical composition of the 2\% Fe-HMS catalysts, energy dispersive spectroscopy (EDS) via X-ray analysis was performed on the samples in the SEM. Table 1 lists a summary of the quantitative EDS results. The EDS results indicate that the ratio of $\mathrm{Fe}(0.34 \%)$ to $\mathrm{Si}$ $(10.68 \%)$ is $3.2 \%($ Ratio $=(0.34 \% / 10.68 \%) \times 100$.$) that is close$ to the synthetic ratio, $2 \%$.

Raman spectroscopy was performed on the CNTs synthesized via CVD incorporating the Fe-HMS catalysts. Analysis of Raman spectra, in Figure 4(a), of the CNTs in aqueous surfactant suspension deposited on an oxidized silicon substrate shows the presence of G- $\left(\sim 1590 \mathrm{~cm}^{-1}\right)$ and D$\left(\sim 1300 \mathrm{~cm}^{-1}\right)$ bands, indicating the graphitic structure of the synthesized nanotubes. The D- and G-bands are understood to be due to the sp3 and sp2 carbon bonding, respectively [13]. The intensity and shape of these peaks give information on the electrical properties of the CNTs. The low D-to-G-band intensity ratio suggests the presence of high-quality, lowdefect CNTs. Also, as can be seen in Figure 4(b), the Raman spectra reveal the presence of several low $\left(200-350 \mathrm{~cm}^{-1}\right)$ Raman-shift peaks. These peaks are understood to be exhibited by the SWNTs due to their radial breathing vibrational modes (RBM) [13]. Using the empirical relationship between the Raman RBM peak frequency $\omega_{\mathrm{RBM}}$ and the SWNT diameter $d_{\text {SWNT }}$ for HiPCO SWNTs, $\omega_{\text {RBM }}=239\left(\mathrm{~cm}^{-1} \mathrm{~nm}\right) / d_{\text {SWNT }}$ 


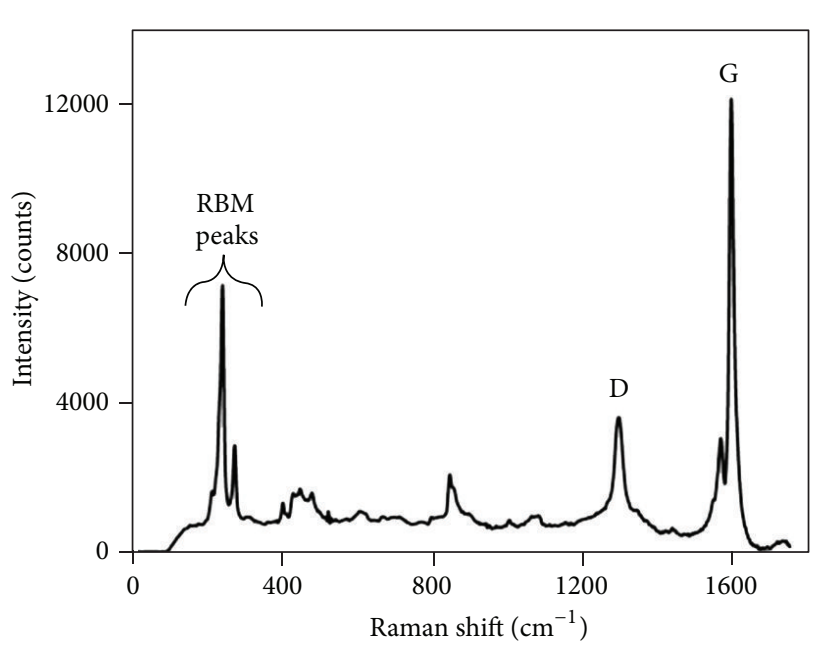

(a)

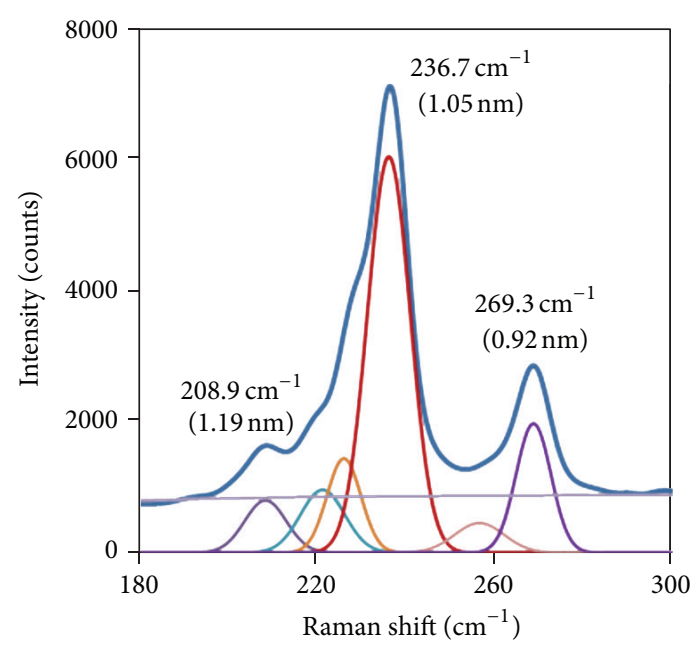

(b)

FIGURE 4: (a) Raman spectrum from carbon nanotubes grown by the novel CVD method incorporating Fe-HMS. (b) The presence of the radial breathing mode (RBM) peaks indicates the presence of single-walled carbon nanotubes (SWNTs).

$(\mathrm{nm})+8.5 \mathrm{~cm}^{-1}$, one can estimate the diameters of the CVD SWNTs exhibiting the RBM spectral peaks $[14,15]$. For example, the RBM peak at $236.7 \mathrm{~cm}^{-1}$ in Figure 4(b) corresponds to an individual nanotube of $1.05 \mathrm{~nm}$ in diameter. Also, from the so-called Kataura plot and the relationship between diameter and chirality, we estimate that the RBM peak originated from the presence of a semiconducting SWNT $[16,17]$. Thus, the Raman spectra characteristics (i.e., the presence of RBM, Dband, and G-band peaks) of the material produced by this novel method are clear evidence not only for the synthesis of carbon nanotubes but also for the specific synthesis of singlewalled carbon nanotubes.

Physical characteristics of the CVD-grown SWNT material were also evaluated by spin-coating an aliquot of the aqueous NaDDBS surfactant suspension of the as-produced SWNTs onto oxidized Si substrates and acquiring topographic images via AFM. AFM observations show that the as-produced CVD SWNT material displayed less surface debris and contaminants compared to depositions of purified HiPCO SWNTs. Since the CVD SWNT material was not subjected to any purification process, the observation indicates that the raw CVD SWNT material has a lower percentage of amorphous carbon and residual catalyst after synthesis than its HiPCO SWNT counterpart. Another possible explanation is that the residue and byproducts of the raw CVD SWNT material are easily dissolved or are not soluble at all in the aqueous surfactant solution used in this study. This property of the as-produced CVD SWNTs minimizes prior processing to purify the nanotube material and reduces the potential damage that can be incurred on CNTs subjected to various purification and chemical processing techniques [18-20].

AFM images also show the presence of small bundles of SWNTs, and typical diameters (as determined from measurements of the height of the bundles) are $3.0-15.0 \mathrm{~nm}$ with measured lengths from 2.0 to $10.0 \mu \mathrm{m}$. In contrast, typical lengths of bundles of similarly processed (in terms of suspension in solvents), purified HiPCO SWNTs are measured in the range of $500 \mathrm{~nm}$ to $3.0 \mu \mathrm{m}$. The longest SWNT bundle observed in one scan is over $20.0 \mu \mathrm{m}$ long with a measured average diameter of $2.0 \pm 0.4 \mathrm{~nm}$. This single nanotube or bundle of nanotubes spans the entire length of the image scan and is shown in Figures 5(b) and 5(c).

Controlling the parameters and the materials used in CVD process can explain the observed long SWNTs. First, choosing methane as the hydrocarbon source in CVD process is one of the key factors because methane is known to be the most kinetically stable hydrocarbon that undergoes the least pyrolytic decomposition at high temperatures. Therefore, the carbon atoms needed for nanotube growth are supplied by the catalytic decomposition of methane on supported iron surfaces. This is one of the primary reasons that the synthesized nanotubes are nearly free of amorphous carbon coatings caused by self-pyrolysis of methane. A second parameter is the CVD reaction time. Limiting the CVD reaction times to only $10 \mathrm{~min}$ at a high methane flow rate prevented the formation of amorphous carbon, producing longer SWNTs, rather than the buildup of carbon as an overcoating to form multiwalled nanotubes (MWCNTs). Thirdly, we find that Fe-HMS as a catalyst produces longer SWNTs due to large domain size (wall thickness) and active nanosize iron centers.

Controlling the deposition and alignment of CNTs onto substrates is often required for precise fabrication of nanotube sensor devices for various applications (strain, temperature, chemical sensing, etc.), and the use of dielectrophoretic assembly of CNTs (both MWNTs and SWNTs) has been investigated previously as a means to accomplish this task [21-25]. To explore the suitability of the CVD SWNTs grown in this study for device fabrication, dielectrophoresis (DEP) is used to attempt controlled deposition of the SWNTs. Briefly, a droplet of the SWNT NaDDBS suspension is placed on the lithographically patterned oxidized silicon substrate containing regions of metal thin film electrodes for concentrated assembly of the CNTs for 10 minutes while an electric field is 


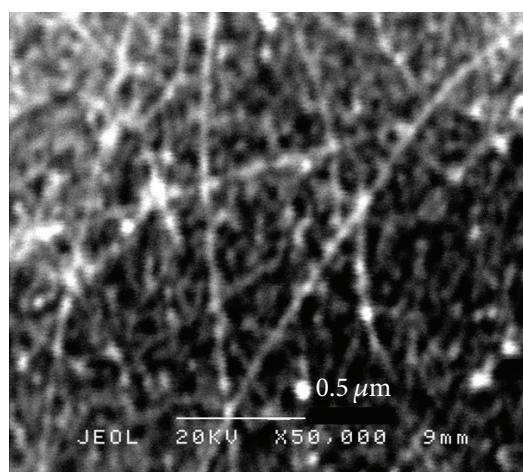

(a)

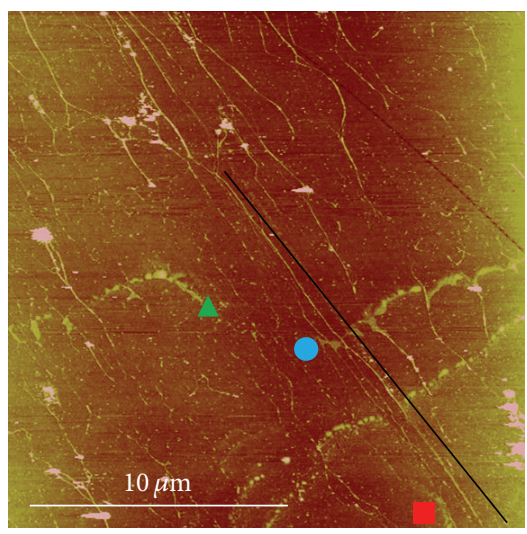

(c)

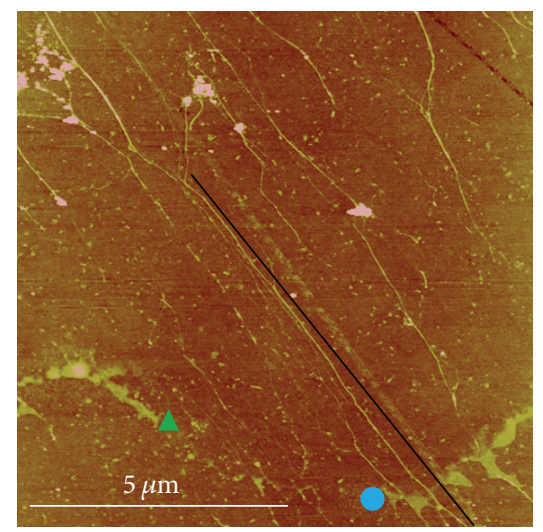

(b)

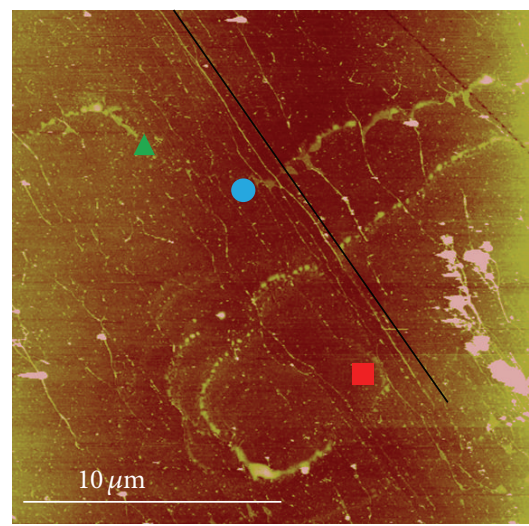

(d)
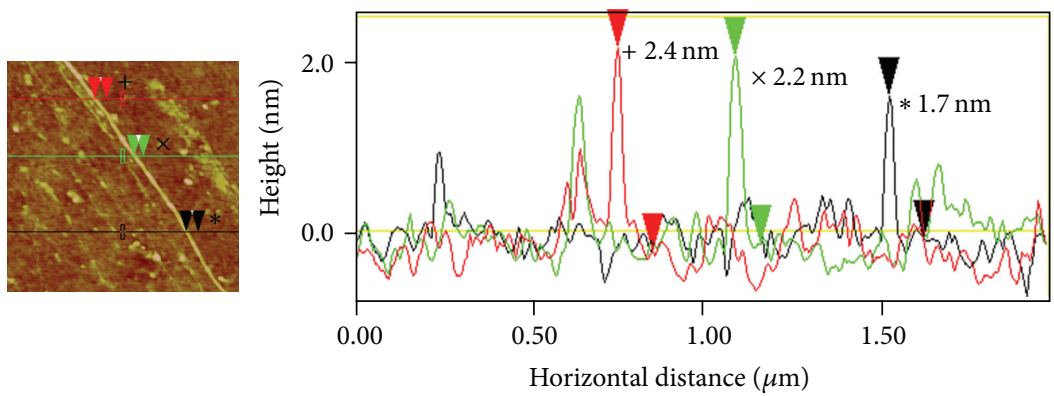

(e)

FIGURE 5: (a) SEM image of as-grown SWNTs on a silicon wafer using the CVD method incorporating Fe-HMS as catalyst. ((b)-(d)) AFM topographic images of as-grown SWNTs in aqueous NaDDBS solution spin-cast onto an oxidized silicon substrate; color range (height scale) in all AFM images is $10 \mathrm{~nm}$. The images show minimal residual catalyst, small-diameter $(3-15 \mathrm{~nm})$ SWNT bundles, and a long ( $>20 \mu \mathrm{m})$ SWNT bundle. The long SWNT bundle is tracked in each image, shown beside the black line as a visual aid. The three markers (triangle, circle, and square) indicate corresponding locations in each image. (e) To determine the diameter of the long SWNT bundle, the height of the bundle was measured at several different positions along the length, yielding an average diameter of $2.0 \pm 0.4 \mathrm{~nm}$.

applied between the electrodes. After the deposition, SWNTs are adhered successfully to the electrodes as observed via AFM. Figure 6 shows an AFM image of the lithographically patterned surface after the CNT deposition. The length of the as-grown CVD SWNTs, combined with their ability to be deposited controllably via DEP, facilitates the insertion of the CVD SWNTs into the design and fabrication of various sensor architectures, similar to previous studies by the authors for CNT strain sensors using HiPCO SWNTs $[26,27]$.

\section{Conclusion}

We have demonstrated a novel approach using Fe-HMS as catalyst in the CVD synthesis of CNTs. Fe-HMS has an ordered nanopore structure in the range of $25 \mathrm{~nm}$ and highly dispersed active iron sites. By combining the Fe-HMS structural properties and controlling the CVD parameters, they produced bundles and individual SWCNTs with observed lengths on the order of several microns. The raw, as-produced SWNT material was able to be dispersed in an aqueous 


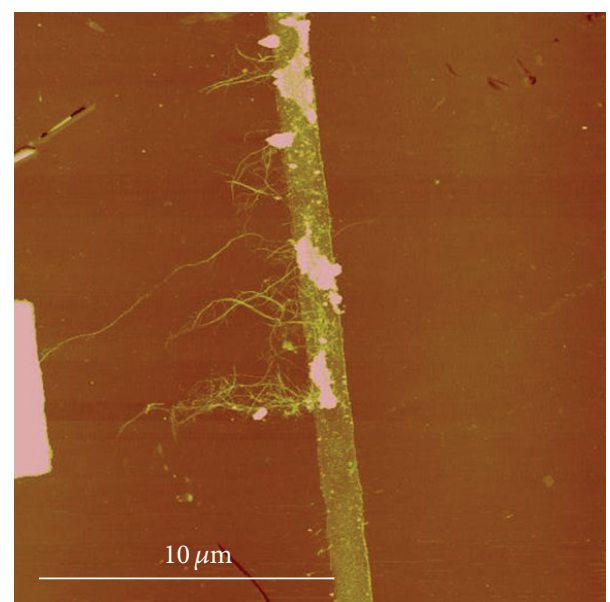

FIGURE 6: AFM image of as-produced SWNTs dispersed in aqueous surfactant solution and deposited via dielectrophoresis (DEP) onto an oxidized Si substrate patterned with metal electrodes; color range (height scale) is $50 \mathrm{~nm}$.

surfactant suspension without any prior purification or processing, and SWNTs deposited from suspension showed minimal surface debris or contamination from residual catalysts. These features make SWNTs synthesized with this CVD method promising candidates for efficient incorporation into polymer composites and for aerospace applications as sensor devices.

\section{Acknowledgments}

The authors would like to thank C. Burkett and B. DeBono for helpful technical contributions to the experiments in this work. This work was partially performed when Dr. Phillip A. Williams held a National Research Council postdoctoral associate appointment at NASA Langley Research Center and Dr. Tarek M. Abdel-Fattah was a NASA Summer Faculty Research Fellow.

\section{References}

[1] M. Meyyappan, Carbon Nanotubes: Science and Applications, Boca Raton, Mass, USA, CRC Press, 2004.

[2] C. Hierold, Carbon Nanotube Devices: Properties, Modeling, Integration and Applications, Wiley-VCH, Weinheim, Germany, 2008.

[3] E. Pop, D. Mann, J. Cao, Q. Wang, K. Goodson, and H. Dai, "Negative differential conductance and hot phonons in suspended nanotube molecular wires," Physical Review Letters, vol. 95, no. 15, Article ID 155505, 2005.

[4] P. Nikolaev, M. J. Bronikowski, R. K. Bradley et al., "Gasphase catalytic growth of single-walled carbon nanotubes from carbon monoxide," Chemical Physics Letters, vol. 313, no. 1-2, pp. 91-97, 1999.

[5] P. T. Tanev and T. J. Pinnavaia, "Mesoporous silica molecular sieves prepared by ionic and neutral surfactant templating: a comparison of physical properties," Chemistry of Materials, vol. 8, no. 8, pp. 2068-2079, 1996.
[6] T. M. Abdel-Fattah, E. J. Siochi, and R. E. Crooks, "Pyrolytic synthesis of carbon nanotubes from sucrose on a mesoporous silicate," Fullerenes Nanotubes and Carbon Nanostructures, vol. 14, no. 4, pp. 585-594, 2006.

[7] P. T. Tanev and T. J. Pinnavaia, "A neutral templating route to Mesoporous molecular sieves," Science, vol. 267, no. 5199, pp. 865-867, 1995.

[8] T. M. Abdel-Fattah and T. J. Pinnavaia, "Tin-substituted mesoporous silica molecular sieve (Sn-HMS): synthesis and properties as a heterogeneous catalyst for lactide ring-opening polymerization," Chemical Communications, no. 5, pp. 665-666, 1996.

[9] M. F. Islam, E. Rojas, D. M. Bergey, A. T. Johnson, and A. G. Yodh, "High weight fraction surfactant solubilization of singlewall carbon nanotubes in water," Nano Letters, vol. 3, no. 2, pp. 269-273, 2003.

[10] J. I. Paredes and M. Burghard, "Dispersions of individual singlewalled carbon nanotubes of high length," Langmuir, vol. 20, no. 12, pp. 5149-5152, 2004.

[11] H. A. Pohl, Dielectrophoresis, the Behavior of Neutral Matter in Nonuniform Electric Fields, Cambridge University Press, Cambridge, Mass, USA, 1978.

[12] M. Dimaki and P. Bøggild, "Frequency dependence of the structure and electrical behaviour of carbon nanotube networks assembled by dielectrophoresis," Nanotechnology, vol. 16, no. 6, pp. 759-763, 2005.

[13] S. Reich, C. Thomsen, and J. Maultzsch, Carbon Nanotubes: Basic Concepts and Physical Properties, Wiley-VCH, Weinheim, Germany, 2004.

[14] H. Kuzmany, W. Plank, M. Hulman et al., "Determination of SWCNT diameters from the Raman response of the radial breathing mode," European Physical Journal B, vol. 22, no. 3, pp. 307-320, 2001.

[15] M. J. O'Connell, Carbon Nanotubes: Properties and Applications, Taylor and Francis, Boca Raton, Mass, USA, 2006.

[16] H. Kataura, Y. Kumazawa, Y. Maniwa et al., "Optical properties of single-wall carbon nanotubes," Synthetic Metals, vol. 103, no. 1-3, pp. 2555-2558, 1999.

[17] J. Maultzsch, H. Telg, S. Reich, and C. Thomsen, "Radial breathing mode of single-walled carbon nanotubes: optical transition energies and chiral-index assignment," Physical Review B, vol. 72, no. 20, Article ID 205438, 2005.

[18] C. A. Furtado, U. J. Kim, H. R. Gutierrez, L. Pan, E. C. Dickey, and P. C. Eklund, "Debundling and dissolution of single-walled carbon nanotubes in amide solvents," Journal of the American Chemical Society, vol. 126, no. 19, pp. 6095-6105, 2004.

[19] M. Monthioux, B. W. Smith, B. Burteaux, A. Claye, J. E. Fischer, and D. E. Luzzi, "Sensitivity of single-wall carbon nanotubes to chemical processing: an electron microscopy investigation," Carbon, vol. 39, no. 8, pp. 1251-1272, 2001.

[20] J. G. Wiltshire, A. N. Khlobystov, L. J. Li, S. G. Lyapin, G. A. D. Briggs, and R. J. Nicholas, "Comparative studies on acid and thermal based selective purification of HiPCO produced singlewalled carbon nanotubes," Chemical Physics Letters, vol. 386, no. 4-6, pp. 239-243, 2004.

[21] M. Dimaki and P. Bøggild, "Waferscale assembly of FieldAligned nanotube Networks (FANs)," Physica Status Solidi A, vol. 203, no. 6, pp. 1088-1093, 2006.

[22] A. H. Monica, S. J. Papadakis, R. Osiander, and M. Paranjape, "Wafer-level assembly of carbon nanotube networks using dielectrophoresis," Nanotechnology, vol. 19, no. 8, Article ID 085303, 2008. 
[23] D. Sickert, S. Taeger, I. Kühne, M. Mertig, W. Pompe, and G. Eckstein, "Strain sensing with carbon nanotube devices," Physica Status Solidi B, vol. 243, no. 13, pp. 3542-3545, 2006.

[24] D. Xu, A. Subramanian, L. Dong, and B. J. Nelson, "Shaping nanoelectrodes for high-precision dielectrophoretic assembly of carbon nanotubes," IEEE Transactions on Nanotechnology, vol. 8, no. 4, pp. 449-456, 2009.

[25] J. Chung and J. Lee, "Nanoscale gap fabrication and integration of carbon nanotubes by micromachining," Sensors and Actuators A, vol. 104, no. 3, pp. 229-235, 2003.

[26] B. Wincheski, M. Namkung, J. Smits, P. Williams, and R. Harvey, "Effect of alignment on transport properties of carbon nanotube/metallic junctions," in Proceedings of the Materials Research Society Symposium Proceedings, pp. 209-214, April 2003.

[27] A. N. Watkins, J. L. Ingram, J. D. Jordan, R. A. Wincheski, J. M. Smits, and P. A. Williams, "Single wall carbon nanotube-based structural health sensing materials," in Proceedings of the NSTI Nanotechnology Conference and Trade Show (NSTI Nanotech '04), vol. 3, pp. 149-152, March 2004. 

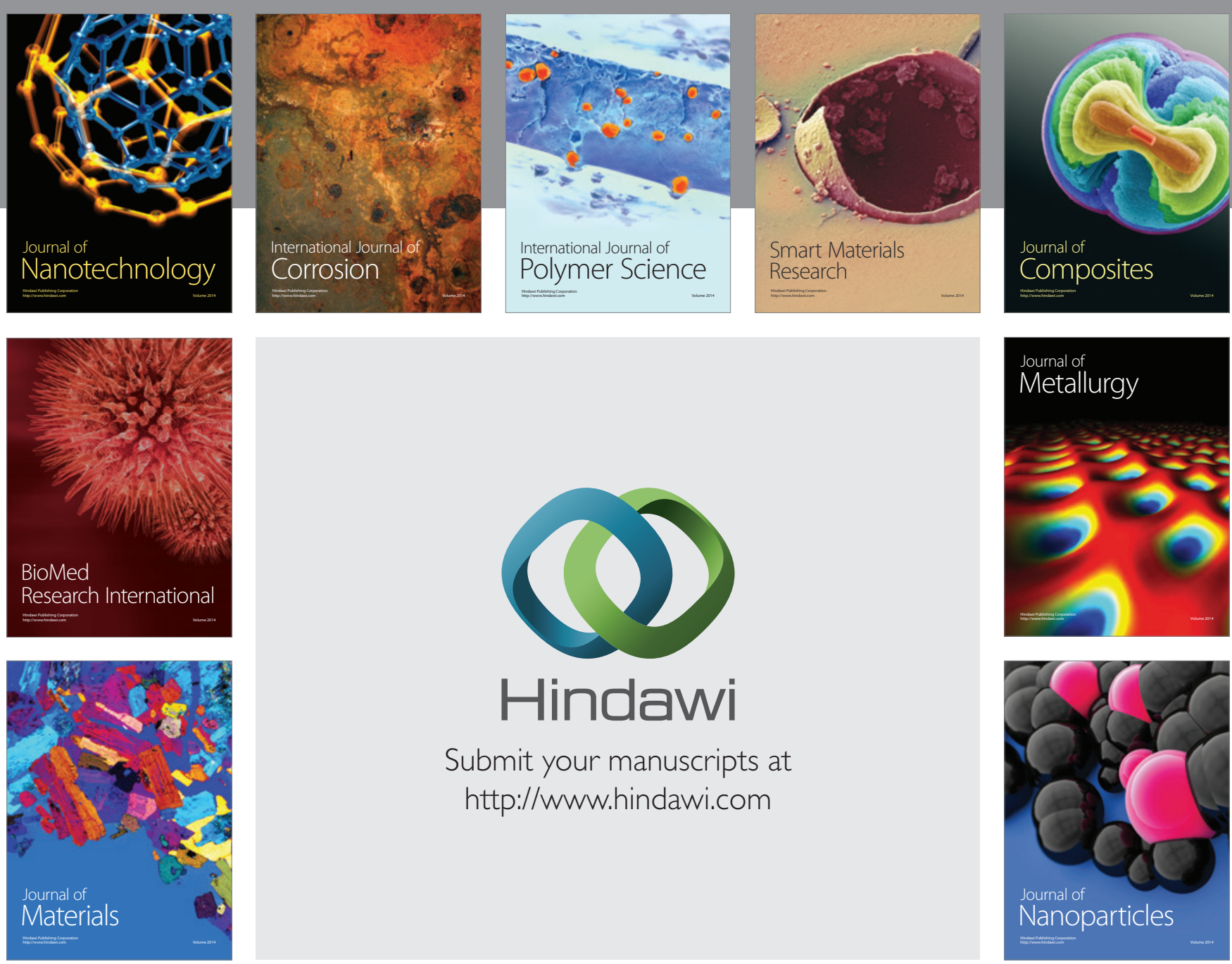

Submit your manuscripts at http://www.hindawi.com
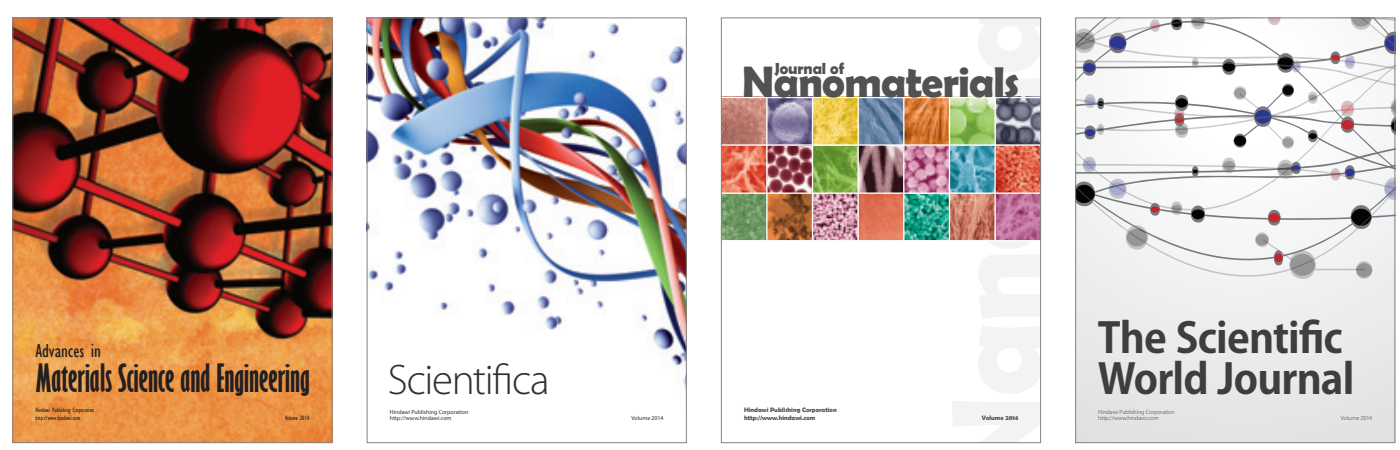

\section{The Scientific World Journal}
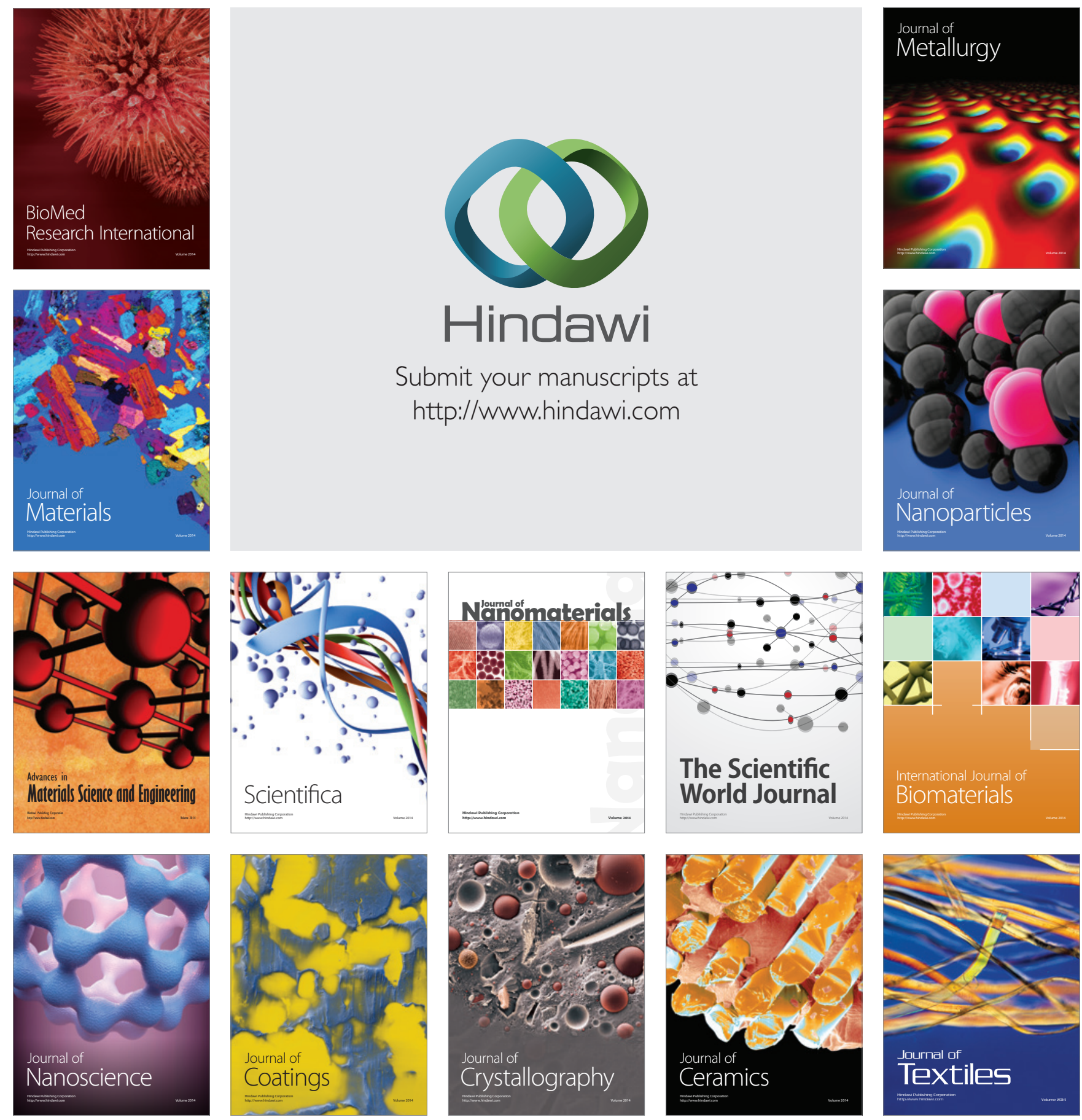